\title{
Forecast of operational data in electric energy plants using adaptive algorithm
}

\author{
Autores \\ Amelec Viloria, Jesús García Guiliany, Hugo Hernandez-P, Luis Cabas Vásquez, \\ Omar Bonerge Pineda Lezama
}

\begin{abstract}
Traditional time series methods offer models whose parameters remain constant over time. However, industrial supply and demand processes require timely decisions based on a dynamic reality. A change in configuration, turning off, or on a production line or process, modifies the problem and the variables to be predicted. Decision support systems must dynamically adapt in order to respond quickly and appropriately to operations and their processes. This methodology is based on obtaining, for each period, the model that best fits the data, evaluating many alternatives and using statistical learning techniques. In this way, the model will adapt to the data in practice and make decisions based on experience. With three months of testing for the estimation of variables associated with supply and demand processes, predictions that differ less than 8 hundredths (less than 0.08 ) or $0.1 \%$ of the measured value were obtained. This indicates that data science and statistical learning represent an important area of research for variable prediction and process optimization.
\end{abstract}

Palabras clave

Time series models, Estimation, Forecasts, Data analysis, Data mining, Statistical learning, Decision trees. 